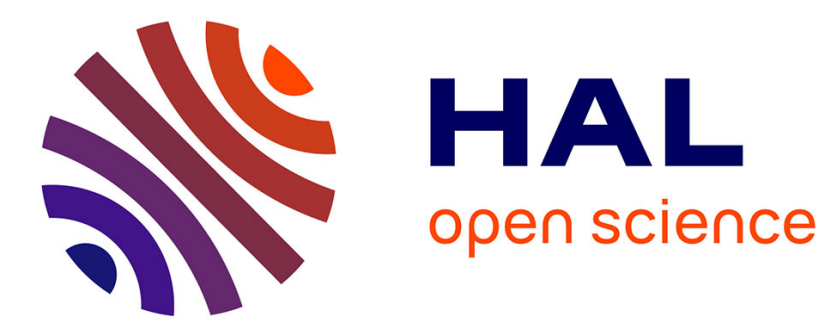

\title{
Electron-acoustic solitons and double layers in the inner magnetosphere
}

\author{
I. Y. Vasko, O. V. Agapitov, F. S. Mozer, J. W. Bonnell, A. V. Artemyev, \\ Vladimir Krasnoselskikh, G. Reeves, G. Hospodarsky
}

\section{- To cite this version:}

I. Y. Vasko, O. V. Agapitov, F. S. Mozer, J. W. Bonnell, A. V. Artemyev, et al.. Electron-acoustic solitons and double layers in the inner magnetosphere. Geophysical Research Letters, 2017, 44 (10), pp.4575-4583. 10.1002/2017GL074026 . insu-01557414

\section{HAL Id: insu-01557414 https://hal-insu.archives-ouvertes.fr/insu-01557414}

Submitted on 6 Jul 2017

HAL is a multi-disciplinary open access archive for the deposit and dissemination of scientific research documents, whether they are published or not. The documents may come from teaching and research institutions in France or abroad, or from public or private research centers.
L'archive ouverte pluridisciplinaire HAL, est destinée au dépôt et à la diffusion de documents scientifiques de niveau recherche, publiés ou non, émanant des établissements d'enseignement et de recherche français ou étrangers, des laboratoires publics ou privés. 


\section{Geophysical Research Letters}

\section{RESEARCH LETTER \\ 10.1002/2017GL074026 \\ Electron-acoustic solitons and double layers in the inner magnetosphere}

Key Points:

- Electrostatic solitary waves with asymmetric bipolar parallel electric field contribute to broadband wave activity in inner magnetosphere

- Asymmetric ESW follow KdV dispersion relation and have spatial scales comparable to those of electron-acoustic solitons

- Asymmetric ESW are interpreted in terms of electron-acoustic solitons and electron-acoustic shock waves

Supporting Information: - Supporting Information S1

Correspondence to:

I. Y. Vasko,

vaskoiy@gmail.com

\section{Citation:}

Vasko, I. Y., O. V. Agapitov, F. S. Mozer, J. W. Bonnell, A. V. Artemyev, V. V. Krasnoselskikh, G. Reeves, and G. Hospodarsky (2017), Electron-acoustic solitons and double layers in the inner magnetosphere, Geophys. Res. Lett., 44, 4575-4583, doi:10.1002/2017GL074026.

Received 1 MAY 2017 Accepted 13 MAY 2017 Accepted article online 16 MAY 2017 Published online 29 MAY 2017

○2017. American Geophysical Union. All Rights Reserved.

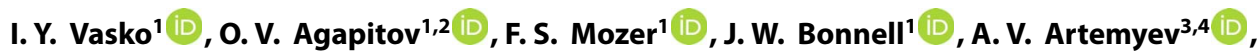 \\ V. V. Krasnoselskikh ${ }^{5}$, G. Reeves ${ }^{6}$, and G. Hospodarsky7 \\ ${ }^{1}$ Space Sciences Laboratory, University of California, Berkeley, California, USA, ${ }^{2}$ Astronomy and Space Physics Department, \\ Taras Shevchenko National University of Kiev, Kiev, Ukraine, ${ }^{3}$ Institute of Geophysics and Planetary Sciences, University of \\ California, Los Angeles, California, USA, ${ }^{4}$ Space Research Institute of the Russian Academy of Sciences, Moscow, Russia, \\ ${ }^{5}$ LPC2E, University of Orléans, Orléans, France, ${ }^{6}$ Los Alamos National Laboratory, Santa Fe, New Mexico, USA, ${ }^{7}$ Department \\ of Physics and Astronomy, University of lowa, lowa City, lowa, USA
}

Abstract The Van Allen Probes observe generally two types of electrostatic solitary waves (ESW) contributing to the broadband electrostatic wave activity in the nightside inner magnetosphere. ESW with symmetric bipolar parallel electric field are electron phase space holes. The nature of ESW with asymmetric bipolar (and almost unipolar) parallel electric field has remained puzzling. To address their nature, we consider a particular event observed by Van Allen Probes to argue that during the broadband wave activity electrons with energy above $200 \mathrm{eV}$ provide the dominant contribution to the total electron density, while the density of cold electrons (below a few eV) is less than a few tenths of the total electron density. We show that velocities of the asymmetric ESW are close to velocity of electron-acoustic waves (existing due to the presence of cold and hot electrons) and follow the Korteweg-de Vries (KdV) dispersion relation derived for the observed plasma conditions (electron energy spectrum is a power law between about $100 \mathrm{eV}$ and $10 \mathrm{keV}$ and Maxwellian above $10 \mathrm{keV}$ ). The ESW spatial scales are in general agreement with the KdV theory. We interpret the asymmetric ESW in terms of electron-acoustic solitons and double layers (shocks waves).

\section{Introduction}

The Van Allen Probes observe generally two types of large-amplitude (from tens to hundreds of $\mathrm{mV} / \mathrm{m}$ ) electrostatic solitary waves (ESW, also called time domain structures) contributing to the broadband electrostatic wave activity observed in the nightside inner magnetosphere [Mozer et al., 2015; Malaspina et al., 2015]. The ESW with symmetric bipolar parallel electric field are electron phase space holes known by numerous observations in the plasma sheet boundary layer [Matsumoto et al., 1994], reconnection current sheets [Cattell et al., 2005], in the auroral [Ergun et al., 1998] and many other regions [e.g., Mozer et al., 2015]. The critical argument in interpreting the symmetric bipolar ESW as electron phase space holes is a divergent parallel electric field configuration (Figure 1a) and numerous simulations demonstrating electron hole formation in a nonlinear stage of electron streaming-type instabilities [e.g., Omura et al., 1996]. On the contrary, the nature of the ESW with asymmetric bipolar (and almost unipolar) parallel electric field (further called asymmetric ESW) remains puzzling. Interestingly, the asymmetric ESW have a convergent parallel electric field configuration, and a finite potential drop across these ESW may not be ruled out (Figure 1b). The asymmetric ESW (as well as electron holes) have velocities comparable to electron thermal velocities and hence should correspond to some electron plasma mode [Agapitov et al., 2015; Vasko et al., 2015].

ESW observed aboard the S3-3 spacecraft in the auroral region also exhibited symmetric and asymmetric bipolar parallel electric fields, but they propagated with velocities comparable to ion thermal velocities [Temerin et al., 1982; McFadden et al., 2003]. Symmetric ESW were interpreted in terms of ion phase space holes [Hudson et al., 1983] or rarefactive ion-acoustic solitons [Lotko and Kennel, 1983]. Asymmetric ESW were interpreted in terms of ion-acoustic double layers developing due to a spatially asymmetric reflection of current-carrying electrons by symmetric ESW [Chanteur et al., 1983]. A similar mechanism cannot produce the asymmetric ESW from electron holes in the nightside inner magnetosphere, since the number density of reflected ions is negligible due to high velocities of electron holes (electrons are not reflected due to the positive electron hole electrostatic potential). 

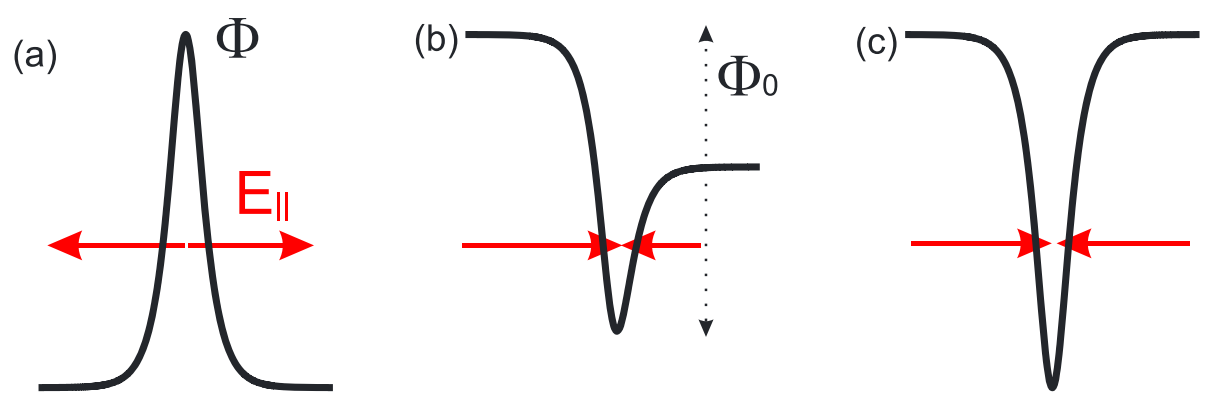

Figure 1. Schematics of parallel electric field configuration of ESW observed in the inner magnetosphere (propagation from right to left): (a) symmetric bipolar divergent parallel electric field (corresponds to electron phase space holes); (b) asymmetric bipolar convergent parallel electric field; (c) symmetric bipolar convergent parallel electric field (corresponds to electron-acoustic solitons; such ideally symmetric ESW are never observed). Black curves are profiles of the electrostatic potential, while red arrows indicate the parallel electric field direction and magnitude. $\Phi_{0}$ is the absolute peak value of the electrostatic potential.

The plasma mode potentially corresponding to the asymmetric ESW is the electron-acoustic mode, which exists in plasmas with a two-temperature electron population [Watanabe and Taniuti, 1977]. The electron-acoustic mode is similar to ion-acoustic mode with the role of ions played by cold electrons. The Landau damping of the electron-acoustic mode depends on fine details of the electron distribution function in the resonant velocity range. In plasmas with Maxwellian cold and hot electrons the conditions of weak Landau damping are $n_{c} \lesssim n_{h}$ and $T_{h} \gtrsim 10 T_{c}$ (see for details, Gary and Tokar [1985]). Electron-acoustic waves propagating along the background magnetic field have a dispersion law $\omega \approx k v_{0}\left(1-k^{2} d^{2} / 2\right)$ similar to ion-acoustic waves [Sagdeev, 1966], where in a plasma with Maxwellian cold and hot electrons the electron-acoustic velocity $v_{0}$ and the dispersive scale $d$ are

$$
v_{0}=\sqrt{\frac{T_{h}}{m_{e}}}\left(\frac{n_{c}}{n_{h}}+3 \frac{T_{c}}{T_{h}}\right)^{1 / 2}, \quad d=\left(\frac{T_{h}}{4 \pi e^{2} n_{h}}\right)^{1 / 2}\left(1+3 \frac{T_{c}}{T_{h}} \frac{n_{h}}{n_{c}}\right)^{-1 / 2}
$$

and $T_{c}$ and $T_{h}$ are cold and hot electron temperatures and $-e$ and $m_{e}$ are electron charge and mass [e.g., Mace et al., 1991]. This dispersion law makes the existence of electron-acoustic solitons and shock waves (double layers) possible [e.g., Sagdeev, 1966; Mace et al., 1991; Cattaert et al., 2005]. Interestingly, to date, there has been no direct identification of electron-acoustic solitons and double layers in observations.

In this letter we select one particular event of Van Allen Probe observations of the broadband electrostatic wave activity and corresponding asymmetric ESW in the inner magnetosphere. We demonstrate that during the broadband wave activity the electron population consists of cold and hot electrons with $T_{h} \gg T_{c}$ and $n_{c} \ll n_{h}$. Based on analysis of the ESW velocities, spatial scales, and electric field configuration, we interpret the asymmetric ESW in terms of electron-acoustic solitons (distorted by electron reflection) and double layers.

\section{Observations}

We consider an event observed by Van Allen Probe B on 13 November 2012, and we use data provided by the following instruments: Electric Field and Waves (EFW) instrument [Wygant et al., 2013], Electric and Magnetic Field Instrument Suite and Integrated Science (EMFISIS) [Kletzing et al., 2013], and Helium, Oxygen, Proton, and Electron (HOPE) detector [Funsten et al., 2013].

Figure $2 \mathrm{~b}$ presents a spectrum of electric field fluctuations measured by EMFISIS on the nightside at $L \sim 6$, at magnetic latitude $\lambda \sim-16^{\circ}$, and around $5 \mathrm{~h}$ magnetic local time. The bursts of the broadband electrostatic wave activity extending to a few hundred hertz are observed for about $1 \mathrm{~h}$. Figure $2 \mathrm{a}$ presents estimates of the total electron number density based on the upper hybrid frequency estimates provided by EMFISIS. Figure $2 \mathrm{a}$ also presents the number density of electrons with energies above $200 \mathrm{eV}$ provided by the HOPE detector. During the entire event the total electron number density is between 2 and $6 \mathrm{~cm}^{-3}$ and electrons with energies above $200 \mathrm{eV}$ frequently provide the dominant contribution. These conditions may seem to be uncommon for the inner magnetosphere, where one could expect cold electrons of ionospheric origin with energies below a few eV to provide a dominant contribution into the total electron number density. 


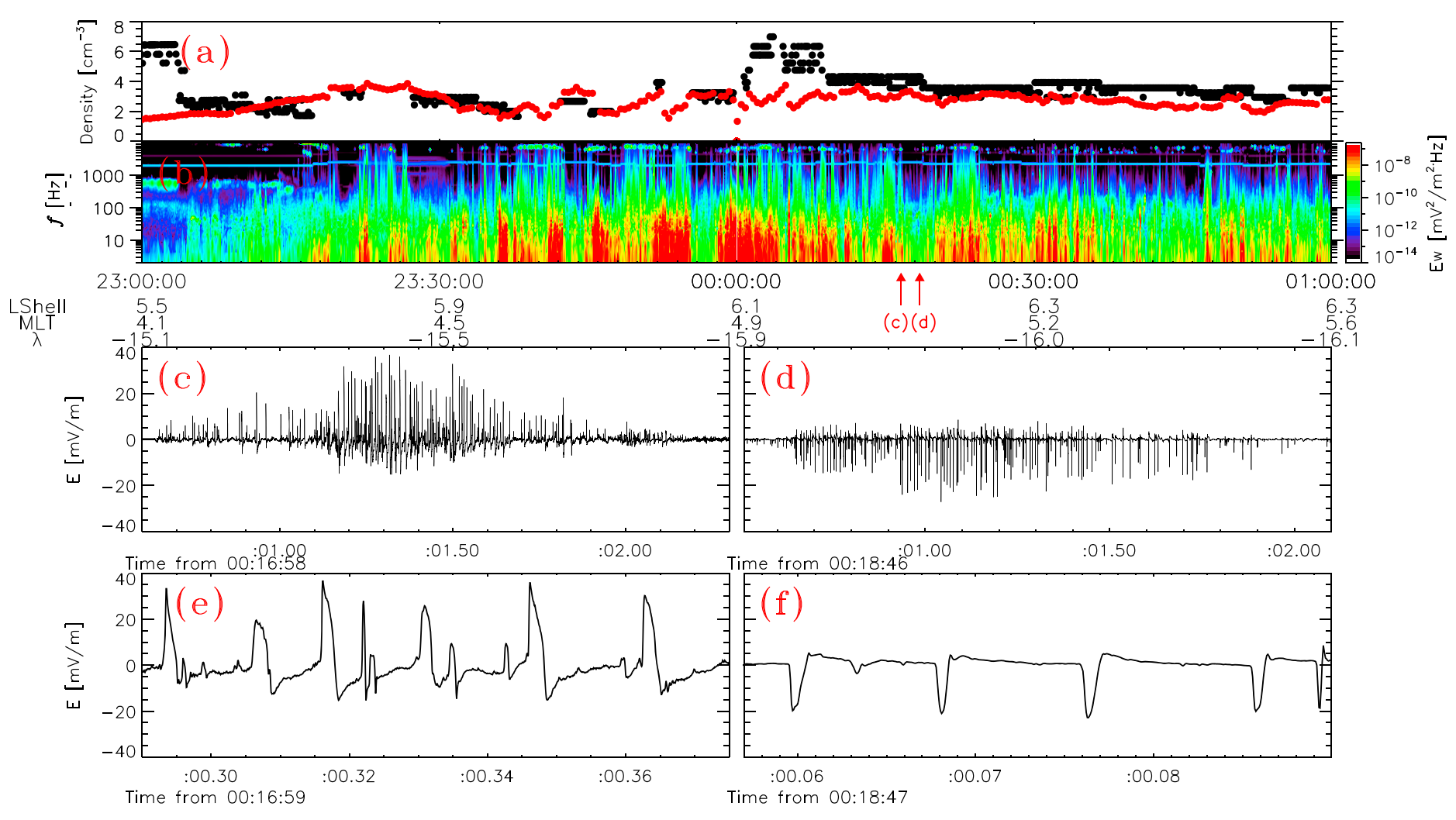

Figure 2. Van Allen Probe B observations on 13 November 2012: (a) the total electron density (black dots) estimate based on EMFISIS measurements of electric field fluctuations at the upper hybrid frequency and the density of electrons with energy above $200 \mathrm{eV}$ (red dots) provided by the HOPE detector; (b) spectrum of electric field fluctuations provided by EMFISIS; $(c, d)$ burst mode $(16,384$ samples/s) electric field measurements provided by the EFW instrument for a few second time intervals around 00:16:58 and 00:18:47 UT indicated with red arrows in Figure $2 \mathrm{~b}$; $(\mathrm{e}, \mathrm{f})$ subintervals of a few tens of milliseconds of burst mode measurements in Figures $2 \mathrm{c}$ and $2 \mathrm{~d}$.

In fact, the broadband electrostatic wave activity is observed either behind particle injection fronts or near the earthward plasma sheet edge [Malaspina et al., 2015] and this explains the dominance of hot electrons [e.g., Gabrielse et al., 2016].

Figures $2 \mathrm{c}$ and $2 \mathrm{~d}$ present high-cadence $(16,384$ samples/s) measurements of the parallel (magnetic field aligned) electric field for about $1 \mathrm{~s}$ time intervals around 00:16:58 UT and 00:18:47 UT indicated in Figure 2b. Figures $2 \mathrm{e}$ and $2 \mathrm{f}$ provide expanded views of a few tens of millisecond subintervals. In accordance with previous observations [Mozer et al., 2015], the broadband wave activity is due to ESW with electric field amplitudes up to a few tens of $\mathrm{mV} / \mathrm{m}$. Further, we focus on the asymmetric ESW, whose typical examples are presented in Figures 2e and 2f. The perpendicular electric fields of these ESW (not shown) are much smaller than their parallel electric fields (e.g., other examples in Vasko et al. [2015]).

Each ESW in Figure 2e has a positive parallel electric field followed by a smaller negative one. Until the ESW propagation direction is known, one cannot distinguish between divergent and convergent parallel electric field configurations. The interferometric analysis [e.g., Vasko et al., 2015] shows that the ESW in Figure 2e propagate in the direction opposite to the background magnetic field (from the equator to higher latitudes) and hence have a convergent parallel electric field configuration presented in Figure 1b, where a finite net potential drop is not ruled out (note that in this schematics the ESW propagate from right to left). In contrast to the ESW in Figure 2e, each ESW in Figure $2 f$ has a negative parallel electric field followed by a much smaller positive one. The interferometric analysis shows that these ESW propagate in the direction opposite to the ESW in Figure 2e. Therefore, these ESW have the same convergent configuration with likely smaller overshoots in Figure $1 \mathrm{~b}$ because of more unipolar parallel electric fields.

We use the interferometric analysis to estimate velocities $V$ of the ESW presented in Figures $2 c$ and $2 d$ (with uncertainty of less than $20 \%$ for $V \lesssim 5000 \mathrm{~km} / \mathrm{s}$ [Vasko et al., 2015]). The ESW propagate almost along 

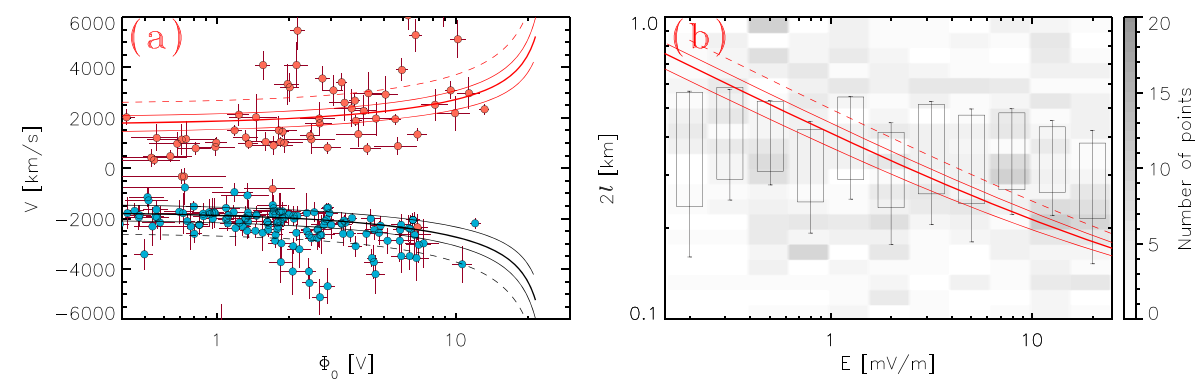

Figure 3. Properties of the ESW presented in Figures $2 \mathrm{c}$ and $2 \mathrm{~d}$ (blue and red dots, respectively): (a) ESW velocity $V$ versus absolute peak value $\Phi_{0}$ of the ESW electrostatic potential. The uncertainties of $V$ and corresponding uncertainties of $\Phi_{0}$ are indicated with error bars. (b) ESW spatial widths 2 / versus absolute peak value $E_{\max }$ of the ESW electric field: the $\left(2 I, E_{\max }\right)$ plane is divided into bins, and the number of ESW in each bin is presented. Each rectangle represents one standard deviation around the average width calculated for ESW with similar $E_{\max }$ (two standard deviation interval is indicated with error bars). The solid black and red curves in Figures $3 \mathrm{a}$ and $3 \mathrm{~b}$ represent theoretical $\mathrm{KdV}$ dispersion relations $V$ versus $\Phi_{0}$ and $D$ versus $E_{\max }$ corresponding to different cold to total electron density ratio, $n_{c} / n_{0}=0.05$, $0.075,0.1$, and 0.15 (the dispersion relations for $n_{c} / n_{0}=0.15$ is indicated with dashed curves).

the magnetic field lines. We also estimate the absolute peak value $\Phi_{0}$ of the ESW electrostatic potential (indicated in Figure 1b) by integrating the dominant unipolar part of the ESW parallel electric field $\Phi_{0}=$ $-\int E_{\|} V \mathrm{~d} t$, where $V$ is positive or negative for ESW propagating along or opposite to the background magnetic field, respectively. For each ESW the dominant unipolar part of the ESW electric field is fitted to the Gaussian model $E_{\|}=E_{\max } \exp \left(-V^{2} t^{2} / 2 l^{2}\right)$ and the best fit half width $/$ is estimated.

Figure 3a presents the ESW velocities and absolute peak values of the ESW electrostatic potential. The ESW presented in Figures $2 \mathrm{c}$ and $2 \mathrm{~d}$ are indicated with blue and red dots, and except for a few cases (noticeable in Figure 3a) they propagate opposite to and along the background magnetic field, respectively. The observed ESW have electrostatic potential peak values below $10 \mathrm{~V}$ and velocities in the range from about 1000 to $5000 \mathrm{~km} / \mathrm{s}$. Figure 3a shows that ESW velocities are larger for larger ESW potential peak values. Figure 3b presents ESW distribution over the spatial width $2 /$ and absolute peak value $E_{\max }$ of the ESW electric field: the $\left(2 I, E_{\max }\right)$ plane is divided into bins and the number of ESW in each bin is determined. We can see that the

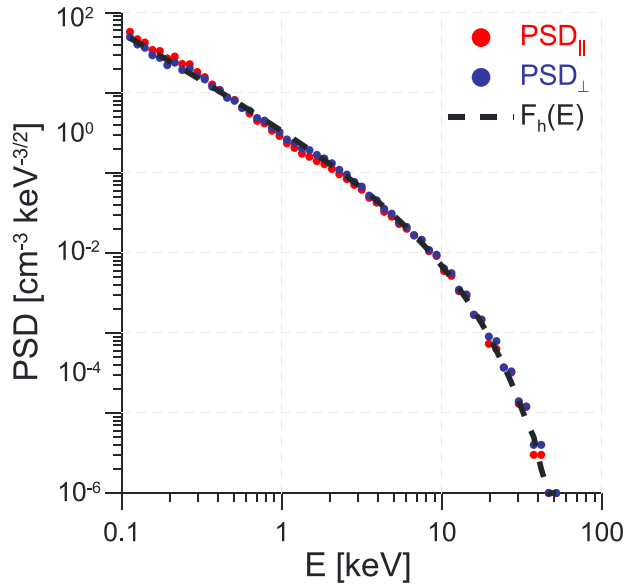

Figure 4. The electron energy spectra (phase space densities) corresponding to averaged parallel (red dots) and perpendicular (blue dots) electron fluxes compiled by the HOPE detector for $1 \mathrm{~min}$ around 00:18:47 UT. The perpendicular flux is averaged over the pitch angle range $\left[60^{\circ}, 120^{\circ}\right]$, while the parallel flux is averaged over the pitch angle ranges $\left[0^{\circ}, 30^{\circ}\right]$ and $\left[150^{\circ}, 180^{\circ}\right]$. The fitting curve (black) corresponds to the model spectrum given by equation (2). The energy spectrum below $100 \mathrm{eV}$ (not shown) is potentially contaminated by photoelectrons and is not included into the fit.
ESW spatial widths are in the range $0.1-1 \mathrm{~km}$. Figure $3 \mathrm{~b}$ also presents the average spatial width for ESW with similar $E_{\max }$ (one and two standard deviations are indicated as well) and demonstrates no clear trend in the spatial width variation with the electric field absolute peak value.

During the earliest period of Van Allen Probes operation (till April 2013) the high-frequency $(>16 \mathrm{~Hz}$ ) electric field measurements of the EFW instrument (providing burst mode data) were AC coupled (Alternating (Capacitive) Coupling), implying that a possible net potential drop across ESW is removed by the electronics. The AC coupling can also distort the original ESW waveform. In the supporting information we present results of the filtering of artificial signals corresponding to series of electrostatic spikes with exactly unipolar parallel electric field. This analysis shows that a "tail" behind each ESW seen in Figure $2 f$ can be a spurious effect due to the electronics. A similar effect is noticeable for the ESW in Figure 2e, but overall, their asymmetric bipolar (rather than 
almost unipolar) electric fields cannot be explained only by the AC coupling and should be real. We conclude that in reality the ESW with convergent electric field can have both asymmetric bipolar and almost unipolar parallel electric fields.

Figure 4 presents electron energy spectra (phase space densities) above $100 \mathrm{eV}$ corresponding to averaged parallel and perpendicular electron fluxes compiled by the HOPE detector for about 1 min around 00:18:47 UT. The energy spectra corresponding to electron fluxes compiled around 00:16:58 UT are similar (not shown). The electron fluxes below about $100 \mathrm{eV}$ (not shown) are potentially contaminated by photoelectrons. The averaged perpendicular flux is calculated by averaging electron fluxes over the pitch angle range $\left[60^{\circ}, 120^{\circ}\right]$, while the average parallel flux is calculated by averaging electron fluxes over the pitch angle ranges $\left[0^{\circ}, 30^{\circ}\right]$ and $\left[150^{\circ}, 180^{\circ}\right]$. Figure 4 shows that the parallel and perpendicular energy spectra are similar, indicating a more or less isotropic electron energy spectrum above $100 \mathrm{eV}$. The spectrum is a power law between about $100 \mathrm{eV}$ and $10 \mathrm{keV}$ and Maxwellian above about $10 \mathrm{keV}$. Interestingly, qualitatively similar electron energy spectra are typical for the near-Earth plasma sheet [e.g., Artemyev et al., 2014]. We fit the observed spectra to the model spectrum

$$
F_{h}(E)=\frac{n_{h}}{G_{0}}\left(\frac{m_{e}}{2 \pi T_{*}}\right)^{3 / 2}\left[1+\frac{E}{\kappa T_{*}}\right]^{-(\kappa+1)} \exp \left[-\frac{E}{E_{0}}\right]
$$

where the normalization constant $G_{0} \equiv G(0)$ and

$$
G(\phi)=\frac{2}{\sqrt{\pi}} \int_{0}^{\infty} \frac{\sqrt{\epsilon} e^{-\epsilon / \epsilon_{0}}}{(1+\phi / \kappa+\epsilon / \kappa)^{\kappa+1}} d \epsilon
$$

where $\epsilon_{0}=E_{0} / T_{*}$. Figure 4 demonstrates that the model spectrum with parameters $\kappa \sim 0.2, E_{0} \sim 8 \mathrm{keV}$, $T_{*} \sim 30 \mathrm{eV}$, and $n_{h} \sim 3.2 \mathrm{~cm}^{-3}$ fits the measured electron energy spectrum above $100 \mathrm{eV}$. The electron number density corresponding to the model spectrum $n_{h} \sim 3.2 \mathrm{~cm}^{-3}$ is in a satisfactory agreement with the density of hot electrons presented in Figure 2a. We stress that the electron temperature determined as the second moment of the electron spectrum (2) is about $T_{h} \sim 2 \mathrm{keV}$ which is much larger than parameter $T_{*} \sim 30 \mathrm{eV}$. This substantial difference appears because of the low power law index $\kappa$, so that the temperature is largely determined by $E_{0}$.

According to Figure 2a the total electron density around 00:18:47 UT is $n_{0} \sim 3.5 \mathrm{~cm}^{-3}$. Although the accuracy of this estimate is not extremely high, it indicates the presence of a cold electron population (or populations) below $100 \mathrm{eV}$ with the number density below a few tenths of the total electron number density. The Van Allen Probes do not allow us to determine the electron energy spectrum below $100 \mathrm{eV}$. However, THEMIS (Time History of Events and Macroscale Interactions during Substorms) spacecraft has recently provided measurements of electron fluxes at energies as low as a few eV with about $1 \mathrm{eV}$ resolution [Mozer et al., 2017]. These measurements confirm that the plasma conditions with a dominant hot electron population can be realized in the nightside inner magnetosphere. They also show that the coldest electron population is Maxwellian with temperature of a few eV in accordance with its ionospheric origin. There can be generally a few electron populations present below $100 \mathrm{eV}$, but in the theoretical model developed in the next section we assume that the electron population consists of cold Maxwellian electrons (with temperature $T_{c}$ of a few eV and density $n_{c}$ below a few tenths of the total electron density) and hot electrons with the energy spectrum (2).

\section{Theoretical Interpretation}

We focus on properties of nonlinear electron-acoustic solitary waves that can propagate under the observed plasma conditions. Based on equation (1), we expect that for $n_{c} \ll n_{h}$ and $T_{c} n_{h} \ll T_{h} n_{c}$ the electron-acoustic velocity is much larger (smaller) than the thermal velocity of cold (hot) electrons. Therefore, in describing electron-acoustic perturbations, cold electrons can be considered in the frame of cold hydrodynamics $\left(T_{c}=0\right)$, while hot electrons should be described using kinetic theory. Since the electron-acoustic velocity is much smaller than the thermal velocity of hot electrons, the inertia of hot electrons can be neglected (this corresponds to neglect of $\partial / \partial t$ in the kinetic equation) and the kinetic equation yields the distribution function $\tilde{F}_{h}=F_{h}(E-e \Phi)$, where $F_{h}(E)$ is arbitrary unperturbed distribution function and $\Phi$ denotes the electrostatic potential of electron-acoustic perturbations. For Maxwellian $F_{h}(E)$ hot electrons are distributed according to the Boltzmann distribution and properties of linear electron-acoustic waves are given by equation (1). 
In our event hot electrons have non-Maxwellian distribution function (2). Therefore, the hot electron density is described by a modified Boltzmann distribution

$$
\tilde{n}_{h}(\phi)=n_{h} \exp \left(-\phi / \epsilon_{0}\right) G(\phi) / G_{0}
$$

where $\phi=-e \Phi / T_{*}$ denotes the normalized electrostatic potential. Since $\epsilon_{0}=E_{0} / T_{*} \sim 250$ the exponential factor can be neglected. Using the method suggested by Karpman [1979], we find that small (but finite) amplitude electron-acoustic perturbations propagating along the background magnetic field (the $Z$ axis) are described by the following equation

$$
\frac{\partial \phi}{\partial t}+v_{0}\left(1+\frac{3+\gamma}{2 \beta} \phi\right) \frac{\partial \phi}{\partial z}+\frac{1}{2} v_{0} d^{2} \frac{\partial^{3} \phi}{\partial z^{3}}=-\frac{\beta n_{0} v_{0}}{2 n_{c}} \frac{\partial N_{r}[\phi]}{\partial z}
$$

where $\beta=n_{c} G_{0} / n_{h}\left|G_{0}^{\prime}\right|$ and $\gamma=n_{c} G_{0} G_{0}^{\prime \prime} / n_{h}\left|G_{0}^{\prime}\right|^{2}$ are numerical factors appearing due to expansion of the hot electron density (4) up to terms of the second order in $\phi$ (primes denote derivatives with respect to the argument, e.g., $\left.G_{0}^{\prime}=(\mathrm{d} G / \mathrm{d} \phi)_{\phi=0}\right)$ and parameters

$$
v_{0}=\sqrt{\frac{T_{s}}{m_{e}}}\left(\frac{n_{c}}{n_{h}}\right)^{1 / 2}, \quad d=\left(\frac{T_{s}}{4 \pi e^{2} n_{h}}\right)^{1 / 2}
$$

are determined by "temperature" $T_{s}$ proportional to compressibility of hot electrons

$$
T_{s}=\left(\frac{\partial \tilde{p}_{h}}{\partial \tilde{n}_{h}}\right)_{\phi=0}=\left(\frac{\partial \tilde{p}_{h} / \partial \phi}{\partial \tilde{n}_{h} / \partial \phi}\right)_{\phi=0}=T_{*} \frac{G_{0}}{\left|G_{0}^{\prime}\right|}
$$

where the hot electron pressure $\tilde{p}_{h}(\phi)$ satisfies hydrodynamic pressure balance $\nabla \tilde{p}_{h}=-T_{*} \tilde{n}_{h}(\phi) \nabla \phi$ so that $\left(\partial \tilde{p}_{h} / \partial \phi\right)_{\phi=0}=-T_{*} n_{h}$.

The right-hand side of equation (5) includes some nonlocal functional $N_{r}[\phi]$ that we temporally assume to be zero. Then equation (5) is the famous Korteweg-de Vries (KdV) equation [e.g., Mace et al., 1991], where the nonlinear term describes steepening, while the last term describes dispersion at scales smaller than the dispersive scale. For $\kappa \gg 1$ the distribution function (2) becomes Maxwellian and the KdV equation (5) coincides with the one derived by Mace et al. [1991] for Maxwellian hot electrons (note that for $\kappa \gg 1$ we have $G(\phi)=e^{-\phi}$ and hence $G_{0}=\left|G_{0}^{\prime}\right|=G_{0}^{\prime \prime}=1$ ). Neglecting the nonlinear term in equation (5), we obtain the dispersion law for linear electron-acoustic waves $\omega=k v_{0}\left(1-k^{2} d^{2} / 2\right)$, which confirms that $v_{0}$ and $d$ defined by equation (6) are electron-acoustic velocity and dispersive scale in the case of the non-Maxwellian distribution (2) of hot electrons. These parameters are determined by "temperature" $T_{s}$ that differs from $T_{*} \sim 30 \mathrm{eV}$ by a factor $G_{0} /\left|G_{0}^{\prime}\right|$. For the observed distribution function ( $\kappa \sim 0.2$ and $\epsilon_{0} \sim 250$ ) we find that $T_{s} \sim 3.5 T_{*} \sim 100 \mathrm{eV}$. We stress that due to the non-Maxwellian electron spectrum, temperature $T_{s}$ is also different from the true hot electron temperature $T_{h} \sim 2 \mathrm{keV}$ (the second moment of the distribution function). For $\kappa \gg 1$ temperature $T_{s}$ and the true electron temperature $T_{h}$ would be both equal to $T_{*}$. Because we have $T_{c} n_{h} \ll T_{s} n_{c}$, the neglect of finite cold electron temperature effects is justified.

In physical units the soliton solution of the KdV equation (5) can be written in the form [e.g., Mace et al., 1991]

$$
\Phi=-\Phi_{0} \operatorname{sech}^{2}\left(\frac{z-V t}{D}\right)
$$

where $\Phi_{0}$ is the absolute peak value of the electrostatic potential, $V$ and $D$ are soliton velocity and spatial width, while the electric field amplitude is $E_{\max } \approx 0.8 \Phi_{0} / D$. Electron-acoustic solitons have symmetric bipolar parallel electric field with a convergent configuration illustrated in Figure 1c (electrostatic potential is negative). The processes resulting in distortion of the soliton symmetric bipolar electric field are discussed later. Meanwhile, we note that the soliton parameters are related to each other through the well-known dispersion relations [e.g., Mace et al., 1991]

$$
V=v_{0}\left(1+\frac{3+\gamma}{6 \beta} \frac{\Phi_{0}}{T_{h}}\right), \quad D=d\left(\frac{12 \beta}{3+\gamma} \frac{T_{h}}{\Phi_{0}}\right)^{1 / 2}
$$

We use the latter dispersion relation rewritten in terms of $D$ and $E_{\max }$. These dispersion relations depend on the cold to total electron density ratio $n_{c} / n_{0}$ that is below a few tenths but not known exactly. The dispersion relations $V$ versus $\Phi_{0}$ and $D$ versus $E_{\max }$ calculated for $n_{c} / n_{0}=0.05,0.075,0.1$, and 0.15 are superimposed on the experimental data in Figure 3. Figure 3a demonstrates that the observed trend of higher ESW velocities 
for larger absolute peak values of the electrostatic potential is reproduced by the KdV dispersion relation. Figure $3 \mathrm{~b}$ demonstrates that the ESW spatial widths $2 I \sim 0.1-1 \mathrm{~km}$ are in general agreement with the spatial widths $D$ predicted by the KdV theory for electron-acoustic solitons. We stress that $D$ is compared with $2 I$ rather than I, since in the previous section we have fitted a dominant unipolar part of the weakly bipolar ESW electric field to the Gaussian model (this introduces the factor of about 2). There is no clear trend of 2 / variation with $E_{\max }$ for the observed ESW that is presumably due to the process resulting in evolution of the soliton symmetric bipolar electric field into asymmetric one.

The processes resulting in distortion of the soliton symmetric bipolar electric field or formation of more or less unipolar electric field are well known in the theory of collisionless shock waves (originally considered for ion-acoustic and magnetosonic solitons) [Sagdeev, 1966]. The KdV equation describes only hydrodynamic effects (steepening and dispersion) and does not describe effects of electrons reflected by a negative soliton electrostatic potential. This kinetic effect is described by the nonlocal functional $N_{r}[\phi]$ on the right-hand side of equation (5). In essence, it represents the density of reflected electrons, while $\partial N_{r} / \partial z$ describes the asymmetry in density of reflected electrons with respect to the peak of the soliton electrostatic potential [Karpman, 1979]. In a current-carrying plasma the electron reflection is asymmetric and results in the development of some local potential drop across a soliton making the soliton electric field asymmetric [e.g., Chanteur et al., 1983; Lynov, 1983]. In the presence of an external potential drop (boundary conditions kept by a larger-scale process) the formation of electron-acoustic shocks with almost unipolar electric field becomes possible [Sagdeev, 1966]. This occurs due to steepening of initial electron-acoustic perturbation that is arrested at some moment by dispersion (at the scale comparable to the dispersive scale) and is kept from disruption into solitons by reflected particles (effective dissipation). According to equation (5) the steepening occurs locally, where $\partial \phi / \partial z<0$, and hence, for shocks propagating along the $Z$ axis we should have $\partial \Phi / \partial z>0$ at their fronts (we recall that $\phi=-e \Phi / T_{*}$ ). This is actually the case for the observed asymmetric ESW as can be seen from schematics in Figure $1 b$ (we recall that ESW propagate from right to left).

Strictly speaking, the KdV equation (5) describes electron-acoustic perturbations with amplitudes $\Phi_{0} / \kappa T_{*} \ll 1$, so that the asymptotic expansion of the hot electron density (4) could be justified. We note that $\kappa T_{*} \sim 5 \mathrm{eV}$ and for some of the observed ESW $\Phi_{0}$ can be as large as $10 \mathrm{~V}$, so that $\Phi_{0} / \kappa T_{*}$ can be of the order of one. In such cases the KdV theory is at the threshold of its applicability, but this is not of principal importance. The models of electron-acoustic solitons with no restriction on their amplitude could be developed using the standard pseudopotential method (dispersion relations are not significantly changed) [e.g., Mace et al., 1991], while the physics of the processes resulting in distortion of the soliton bipolar electric field and formation of shocks remains unchanged.

In summary, the asymmetric ESW have dispersion relation and spatial scales similar to those for electronacoustic solitons. Their convergent electric field configuration is in agreement with what we expect for electron-acoustic solitons (distorted by reflected electrons) and electron-acoustic shocks (formed due to steepening). In addition, the electron-acoustic mode seems to be the only suitable candidate corresponding to the observed asymmetric ESW, since there are no other electrostatic electron modes (with similar phase velocities and spatial scales) to be considered for alternative interpretation. Based on these arguments we interpret the asymmetric ESW in terms of electron-acoustic solitons and shocks.

\section{Discussion and Conclusions}

Electron holes are established to produce the broadband electrostatic wave activity in numerous regions of the near-Earth space [e.g., Matsumoto et al., 1994; Ergun et al., 1998]. Van Allen Probe observations have shown that the broadband wave activity in the inner magnetosphere is due to electron holes (symmetric ESW) and one additional type of ESW with asymmetric parallel electric field [Mozer et al., 2015; Malaspina et al., 2015]. These asymmetric ESW are observed in packets with duration of about $1 \mathrm{simplying}$ that they occupy a region of a few thousand kilometers along the magnetic field propagating from the equator to higher latitudes or in the opposite direction.

In this letter we have addressed the nature of the asymmetric ESW. Using simultaneous EMFISIS and HOPE measurements for a particular event we have shown that the electron population consists of cold and hot electrons with hot electrons providing the dominant contribution to the total electron density. This event is not exceptional. We have checked other events presented in Vasko et al. [2015] and Agapitov et al. [2015] and found similar dominance of the hot electron density and similar electron energy spectra 
(power law spectrum below about $10 \mathrm{keV}$ and Maxwellian above $10 \mathrm{keV}$ ). Based on the theoretical model describing weakly nonlinear electron-acoustic waves, we have interpreted the asymmetric ESW in terms of electron-acoustic solitons and shocks (double layers).

The mechanisms of generation of these ESW have not been thoroughly addressed yet. Fu et al. [2016] have recently reported a negative result in their attempt to simulate generation of electron-acoustic double layers. However, these simulations have been carried out for a narrow range of initial conditions. The mechanism of electron-acoustic shock formation invokes common effects of steepening, dispersion, and particle reflection, but it is sensitive to initial conditions (e.g., only initial perturbations with $\partial \Phi / \partial z>0$ may steepen). We believe that future simulations will succeed in generation of electron-acoustic double layers.

Finally, we point out that electron-acoustic waves were previously suggested as a source of hiss emissions in the dayside polar cusp [Tokar and Gary, 1984] and were directly observed in a source region of the auroral kilometer radiation [Pottelette et al., 1999]. The broadband electrostatic wave activity in the dayside auroral region was suggested to be due to electron-acoustic solitons [Dubouloz et al., 1993]. Our analysis is seemingly the first direct identification of electron-acoustic solitons and double layers, in which existence was predicted rather long ago [Watanabe and Taniuti, 1977]. Thus, Van Allen Probes observations add one additional type of double layers that can be realized in the space plasma [Andersson and Ergun, 2012].

\section{Acknowledgments}

The work of I.V., O.A., F.M., and J.B. was supported by JHU/APL contract 922613 (RBSP-EFW). A.A. acknowledges the support of the Russian Science Foundation (grant 14-12-00824). I.V. thanks Didier Mourenas and Ilan Roth for helpful comments. All data used in this paper can be found at RBSP/EFW database (http://www.space.umn. edu/missions/rbspefwhomeuniversity-of-minnesota), RBSP/ECT database (https://rbsp-ect.lanl.gov), and RBSP/EMFISIS database (http://emfisis.physics.uiowa.edu).

\section{References}

Agapitov, O. V., V. Krasnoselskikh, F. S. Mozer, A. V. Artemyev, and A. S. Volokitin (2015), Generation of nonlinear electric field bursts in the outer radiation belt through the parametric decay of whistler waves, Geophys. Res. Lett., 42, 3715-3722, doi:10.1002/2015GL064145

Andersson, L., and R. E. Ergun (2012), The search for double layers in space plasmas, in Auroral Phenomenology and Magnetospheric Processes: Earth And Other Planets, Geophys. Monogr. Ser., vol. 197, edited by A. Keiling et al., AGU, Washington, D. C., doi:10.1029/2011GM001170.

Artemyev, A. V., A. P. Walsh, A. A. Petrukovich, W. Baumjohann, R. Nakamura, and A. N. Fazakerley (2014), Electron pitch angle/energy distribution in the magnetotail, J. Geophys. Res. Space Physics, 119, 7214-7227, doi:10.1002/2014JA020350.

Cattaert, T., F. Verheest, and M. A. Hellberg (2005), Potential hill electron-acoustic solitons and double layers in plasmas with two electron species, Phys. Plasmas, 12(4), 042901, doi:10.1063/1.1868733.

Cattell, C., et al. (2005), Cluster observations of electron holes in association with magnetotail reconnection and comparison to simulations, J. Geophys. Res., 110, A01211, doi:10.1029/2004JA010519.

Chanteur, G., J. C. Adam, R. Pellat, and A. S. Volokhitin (1983), Formation of ion-acoustic double layers, Phys. Fluids, 26, 1584-1586, doi:10.1063/1.864293.

Dubouloz, N., R. A. Treumann, R. Pottelette, and M. Malingre (1993), Turbulence generated by a gas of electron acoustic solitons, J. Geophys. Res., 98, 17,415-17,422, doi:10.1029/93JA01611.

Ergun, R. E., et al. (1998), FAST satellite observations of large-amplitude solitary structures, Geophys. Res. Lett., 25, 2041-2044, doi:10.1029/98GL00636.

Fu, X., M. M. Cowee, S. P. Gary, and D. Winske (2016), On the generation of double layers from ion- and electron-acoustic instabilities, Phys. Plasmas, 23(3), 032308, doi:10.1063/1.4943881.

Funsten, H. O., et al. (2013), Helium, oxygen, proton, and electron (HOPE) mass spectrometer for the radiation belt storm probes mission, Space Sci. Rev., 179, 423-484, doi:10.1007/s11214-013-9968-7.

Gabrielse, C., C. Harris, V. Angelopoulos, A. Artemyev, and A. Runov (2016), The role of localized inductive electric fields in electron injections around dipolarizing flux bundles, J. Geophys. Res. Space Physics, 121, 9560-9585, doi:10.1002/2016JA023061.

Gary, S. P., and R. L. Tokar (1985), The electron-acoustic mode, Phys. Fluids, 28, 2439-2441, doi:10.1063/1.865250.

Hudson, M. K., W. Lotko, I. Roth, and E. Witt (1983), Solitary waves and double layers on auroral field lines, J. Geophys. Res., 88, 916-926, doi:10.1029/JA088iA02p00916.

Karpman, V. I. (1979), The effects of the interaction between ion-sound solitons and resonance particles in a plasma, Sov. J. Exp. Theor. Phys. 50,695-701.

Kletzing, C. A., et al. (2013), The electric and magnetic field instrument suite and integrated science (EMFISIS) on RBSP, Space Sci. Rev., 179, 127-181, doi:10.1007/s11214-013-9993-6.

Lotko, W., and C. F. Kennel (1983), Spiky ion acoustic waves in collisionless auroral plasma, J. Geophys. Res., 88, 381-394, doi:10.1029/JA088iA01p00381.

Lynov, J. P. (1983), Modification of Korteweg-de Vries solitons in plasmas by resonant particles, Phys. Fluids, 26, 3262-3272, doi:10.1063/1.864101.

Mace, R. L., S. Baboolal, R. Bharuthram, and M. A. Hellberg (1991), Arbitrary-amplitude electron-acoustic solitons in a two-electron-component plasma, J. Plasma Phys., 45, 323-338, doi:10.1017/S0022377800015749.

Malaspina, D. M., J. R. Wygant, R. E. Ergun, G. D. Reeves, R. M. Skoug, and B. A. Larsen (2015), Electric field structures and waves at plasma boundaries in the inner magnetosphere, J. Geophys. Res. Space Physics, 120, 4246-4263, doi:10.1002/2015JA021137.

Matsumoto, H., H. Kojima, T. Miyatake, Y. Omura, M. Okada, I. Nagano, and M. Tsutsui (1994), Electrotastic solitary waves (ESW) in the magnetotail: BEN wave forms observed by GEOTAIL, Geophys. Res. Lett., 21, 2915-2918, doi:10.1029/94GL01284.

McFadden, J. P., C. W. Carlson, R. E. Ergun, F. S. Mozer, L. Muschietti, I. Roth, and E. Moebius (2003), FAST observations of ion solitary waves, J. Geophys. Res., 108, 8018, doi:10.1029/2002JA009485.

Mozer, F. S., O. V. Agapitov, A. Artemyev, J. F. Drake, V. Krasnoselskikh, S. Lejosne, and I. Vasko (2015), Time domain structures: What and where they are, what they do, and how they are made, Geophys. Res. Lett., 42, 3627-3638, doi:10.1002/2015GL063946.

Mozer, F. S., O. A. Agapitov, V. Angelopoulos, A. Hull, D. Larson, S. Lejosne, and J. P. McFadden (2017), Extremely field-aligned cool electrons in the dayside outer magnetosphere, Geophys. Res. Lett., 44, 44-51, doi:10.1002/2016GL072054.

Omura, Y., H. Matsumoto, T. Miyake, and H. Kojima (1996), Electron beam instabilities as generation mechanism of electrostatic solitary waves in the magnetotail, J. Geophys. Res., 101, 2685-2698, doi:10.1029/95JA03145. 
Pottelette, R., R. E. Ergun, R. A. Treumann, M. Berthomier, C. W. Carlson, J. P. McFadden, and I. Roth (1999), Modulated electron-acoustic waves in auroral density cavities: FAST observations, Geophys. Res. Lett., 26, 2629-2632, doi:10.1029/1999GL900462.

Sagdeev, R. Z. (1966), Cooperative phenomena and shock waves in collisionless plasmas, Rev. Plasma Phys., 4, $23-91$.

Temerin, M., K. Cerny, W. Lotko, and F. S. Mozer (1982), Observations of double layers and solitary waves in the auroral plasma, Phys. Rev. Lett., 48, 1175-1179, doi:10.1103/PhysRevLett.48.1175.

Tokar, R. L., and S. P. Gary (1984), Electrostatic hiss and the beam driven electron acoustic instability in the dayside polar cusp, Geophys. Res. Lett., 11, 1180-1183, doi:10.1029/GL011i012p01180.

Vasko, I. Y., O. V. Agapitov, F. S. Mozer, and A. V. Artemyev (2015), Thermal electron acceleration by electric field spikes in the outer radiation belt: Generation of field-aligned pitch angle distributions, J. Geophys. Res. Space Physics, 120, 8616-8632, doi:10.1002/2015JA021644.

Watanabe, K., and T. Taniuti (1977), Electron-acoustic mode in a plasma of two-temperature electrons, J. Phys. Soc. Jpn., 43, 1819, doi:10.1143/JPSJ.43.1819.

Wygant, J. R., et al. (2013), The electric field and waves instruments on the radiation belt storm probes mission, Space Sci. Rev., 179, 183-220, doi:10.1007/s11214-013-0013-7. 